\title{
Effectiveness of Surgical Release in Patients With Neglected Congenital Muscular Torticollis According to Age at the Time of Surgery
}

\author{
Kyung-Jay Min, MD, Ah-Reum Ahn, MD, Eun-Ji Park, MD, Shin-Young Yim, MD, PhD
}

The Center for Torticollis, Department of Physical Medicine and Rehabilitation, Ajou University School of Medicine, Suwon, Korea

Objective To identify the correlation between change in spinal deformities after surgical release and age at the time of surgery, and the effectiveness of surgical release in patients with neglected congenital muscular torticollis (CMT).

Methods This was a retrospective study of 46 subjects with neglected CMT who had undergone surgical release at age $\geq 5$ years at a tertiary medical center between January 2009 and January 2014. Spinal deformities were measured on anteroposterior plain radiographs of the cervical and whole spine, both preoperatively and postoperatively, to assess 3 parameters: cervicomandibular angle (CMA), lateral shift (LS), and Cobb angle (CA). We analyzed the change in spinal deformities after surgical release in consideration of age at the time of surgery. Results The median age at the time of surgery was 12.87 years. All 3 parameters showed significant improvement after surgical release (median values, pre- to post-surgery: CMA, $12.13^{\circ}$ to $4.02^{\circ}$; $\mathrm{LS}, 18.13 \mathrm{~mm}$ to $13.55 \mathrm{~mm}$; CA, $6.10^{\circ}$ to $4.80^{\circ}$; all $\left.\mathrm{p}<0.05\right)$. There was no significant correlation between age at the time of surgery and change in CMA ( $\mathrm{R}=0.145, \mathrm{p}=0.341)$ and $\mathrm{LS}(\mathrm{R}=0.103, \mathrm{p}=0.608)$. However, CA showed significant improvement with increasing age $(\mathrm{R}=0.150, \mathrm{p}=0.046)$.

Conclusion We assessed the correlation between change in spinal deformities after surgical release and age at the time of surgery. We found that that surgical release is effective for spinal deformities, even in older patients. These findings enhance our understanding of the effectiveness and timing of surgical release in patients with neglected CMT.

Keywords Congenital muscular torticollis, Treatment outcome, Surgical procedures

Received April 27, 2015; Accepted July 31, 2015

Corresponding author: Shin-Young Yim

The Center for Torticollis, Department of Physical Medicine and Rehabilitation, Ajou University School of Medicine, 164 Worldcup-ro, Yeongtong-gu, Suwon 16499, Korea

Tel: +82-31-219-5284, Fax: +82-31-219-5209, E-mail: syyim@ajou.ac.kr

@ This is an open-access article distributed under the terms of the Creative Commons Attribution Non-Commercial License (http://creativecommons. org/licenses/by-nc/4.0) which permits unrestricted noncommercial use, distribution, and reproduction in any medium, provided the original work is properly cited.

Copyright $\odot 2016$ by Korean Academy of Rehabilitation Medicine

\section{INTRODUCTION}

Congenital muscular torticollis (CMT) is one of the most common musculoskeletal disorders in children. The reported prevalence ranges from $0.3 \%$ to $2.0 \%$, and can be as high as $3.92 \%$ in neonates [1,2]. CMT is defined as a thickening and/or tightness of the unilateral sternocleidomastoid muscle (SCM) characterized by fibrosis, resulting in a shortening of the SCM and consequent lim- 
ited neck motion [3,4].

Approximately 50\%-70\% of SCM mass resolves spontaneously during the first year of life with minimal residual deficits [5]. If there is a lack of neck motion, early physical therapy such as manual stretching of the neck is necessary. Approximately $90 \%$ of cases can be treated primarily through stretching exercises, although surgical release of the affected SCM is recommended for resistant cases [1]. Without adequate treatment, the limitation in the range of motion caused by persistent shortening of the SCM may lead to complications such as pain, spinal deformities, and craniofacial abnormalities [6-9]. Cervical scoliosis and lateral tilt of the head and neck are common spinal deformities in patients with CMT $[5,6,10]$.

Age at the time of surgery is the most important factor influencing the above complications of resistant CMT. According to previous studies, the best outcomes are obtained when patients undergo surgery between the ages of 1 and 4 years [11-13]. However, for various reasons some CMT patients do not proceed with surgical release even though they meet the clinical criteria. Neglected CMT is stated for those patients who did not undergo operative treatment within adequate time $[14,15]$. There has also been considerable debate over the effectiveness and necessity of invasive surgical release in patients with neglected CMT aged 5 years and older $[13,14]$.

According to previous studies, surgical correction in adults with neglected CMT may result in cosmetic and functional improvements, and relieve pain related to neglected CMT [6]. The effectiveness of surgical release was significant even in patients with neglected CMT aged 15 or older [15]. However, there is still a lack of understanding regarding both the effectiveness of surgical release for spinal deformities in patients with neglected CMT and the correlation between the effectiveness of surgical release and age at the time of surgery.

To the best of our knowledge, no study has previously been carried out on the relationship between age at the time of surgery and how much spinal deformities reside in patients with neglected CMT who underwent surgical release. Thus, the intent of this study was to establish this correlation in a group of neglected CMT patients while also demonstrating the effectiveness of surgical release in treating their spinal deformities.

\section{MATERIALS AND METHODS}

This was a retrospective study carried out at a single tertiary medical center. This research was approved by the Institutional Review Board of Ajou University Medical Center.

\section{Subjects}

Subjects who presented to the Center for Torticollis, Department of Physical Medicine and Rehabilitation, Ajou University Hospital, Suwon, Korea, with the symptoms of abnormal posture of the head and neck from January 2009 to January 2014 and met all of the following inclusion criteria were included in this study: 1) subjects with a diagnosis of CMT, 2) subjects who had undergone surgical release of the affected unilateral SCM for CMT, 3) subjects who were $\geq 5$ years at the time of surgery, and 4) subjects who underwent plain radiographic evaluations of the cervical spine or whole spine, once preoperatively, and again between 6 and 15 months after surgery. In cases where subjects underwent plain radiography more than twice between 6 and 15 months after surgery, the last radiograph was used for evaluation (Fig. 1). Subjects with a previous history of surgical release for CMT and those with a previous history of injection of botulinum toxin into the affected SCM for CMT were excluded from the study.

CMT was diagnosed in subjects with shortening of the unilateral SCM resulting in ipsilateral lateral flexion of the head and/or rotation of the chin toward the contralateral shoulder and one or both of the following: 1) magnetic resonance imaging (MRI) of the neck showing

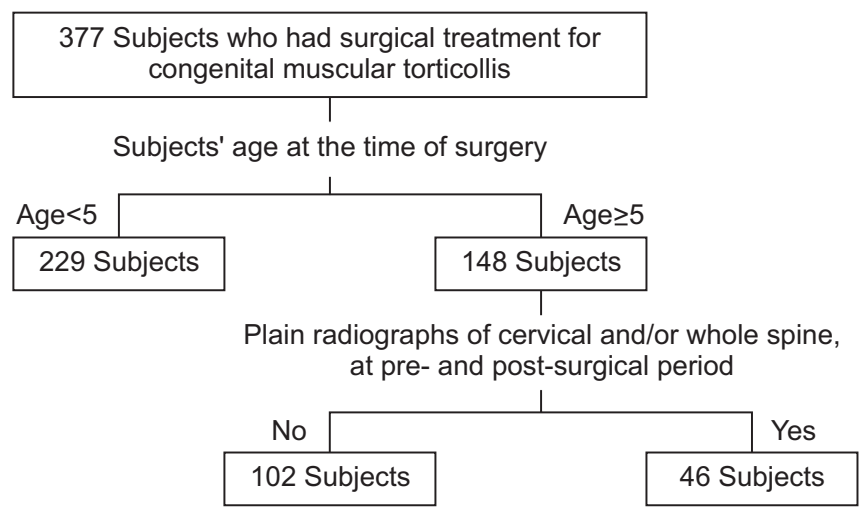

Fig. 1. The algorithm for subject enrollment from January 2009 to January 2014. 
multiple (>2) or gross low-signal intensities (visible to the naked eye) within the SCM [16], 2) the SCM of the involved side was $>2 \mathrm{~mm}$ thicker than that of the contralateral side, as detected by ultrasonography [9].

Surgical release of the SCM for CMT was performed by one surgeon who has extensive experience treating subjects who have CMT. For 2 weeks immediately after surgical release, all patients wore a neck brace to limit motion of the neck to assist with wound healing. Two weeks after surgical release, all patients who undeerwent surgery started rehabilitation exercises. A manual stretching exercise was conducted for patients with a shortened SCM to increase the range of contralateral rotation and ipsilateral lateral flexion of head and neck. Furthermore, strengthening exercises of the head and neck for correction of lateral tilt and trunk stabilization for correction of scoliotic curvatures were conducted. All the rehabilitation exercises were performed three to five times a week by experienced physical therapists and the treatment was ended when a full range of motion for neck rotation was obtained [17].

Acquisition of AP radiographs of cervical spine and whole spine

Anteroposterior (AP) plain radiographs of the cervical spine were taken when subjects showed abnormal posture of the head and neck and/or scoliosis due to neglected CMT. AP plain radiographs of the whole spine were taken when subjects showed vertical displacement of the head or secondary scoliosis due to neglected CMT. The AP plain radiographs were reviewed with Picture Archiving and Communication System (PiViewSTAR ver. 5.0.9.87; Infinitt, Seoul, Korea).

Quantitative analysis of spinal deformities on AP plain radiographs

Quantitative measurements of spinal deformities were made by the first two authors (Min JK and Ahn AR) in an independent and blind fashion. After testing of interrater reliability for quantitative measurement of AP plain radiographs, the mean of the values measured by both authors were used for statistical analysis.

Measurement of the cervicomandibular angle on plain radiograph of the cervical spine

The cervicomandibular angle (CMA) was measured from AP plain radiographs of the cervical spine to evaluate lateral head tilt on the affected side of CMT due to tightness of the affected SCM [10] (Fig. 2A). The CMA was defined as the angle between the line along the upper border of the $\mathrm{C} 7$ spine and the lines connecting the lower borders of both mandibles [18,19] (Fig. 2B).

Measurement of the lateral shift of the head on AP radiographs of the whole spine

The lateral shift (LS) was measured from AP radiographs of the whole spine to evaluate cervical scoliosis with 2 curves produced as the result of spontaneous compensation of neck posture due to lateral head tilt [10] (Fig. 2E). LS was defined as the distance between the vertical line from the center of the sacral upper margin and the central vertical line of the C2 vertebral body [6] (Fig. $2 \mathrm{~F})$.

Measurement of the Cobb angle on AP radiograph of the whole spine

The Cobb angle (CA) was measured from an AP plain radiograph of the whole spine in order to evaluate the magnitude of secondary vertebral scoliosis that had developed to compensate abnormal posture of the head and neck and/or pelvic elevation (Fig. 2I). The CA was defined as the angle formed between a line drawn parallel to the superior endplate of the most cephalad vertebra and a line drawn parallel to the inferior endplate of the most caudal vertebra of the particular curve [20] (Fig. 2J).

\section{The effectiveness of surgical release in neglected CMT}

To prove whether spinal deformities had improved after surgical release in neglected CMT patients, we assessed changes in each parameter after surgical treatment through the Wilcoxon signed-rank test. We considered these changes to be an improvement when postoperative values were lower than those of preoperative period.

Correlation between improvement in spinal deformities and age at the time of surgery

To assess the change in spinal deformities after surgery, we calculated the ratio of the difference between preoperative and postoperative values per preoperative value for CMA, LS, and CA. We refer to these ratios as the CMA ratio, LA ratio, and CA ratio, respectively. These ratios were determined using the following equations. 

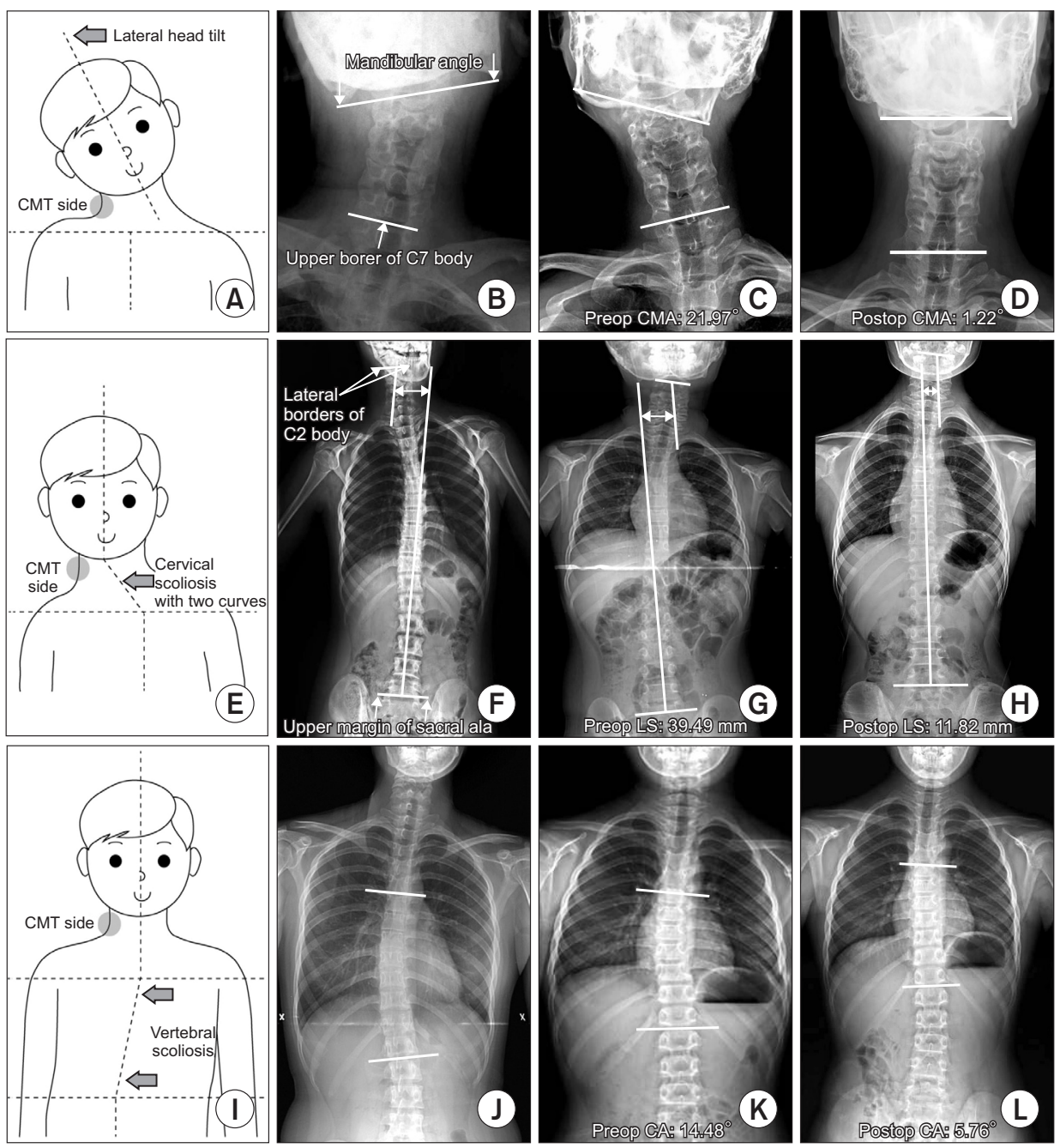

Fig. 2. Spinal deformity measurement in terms of CMA, LS, and $\mathrm{CA}$, and change in spinal deformities after surgical release for neglected CMT. (A) Lateral head tilt toward the affected side of CMT. (B) Measurement of CMA to assess lateral head tilt. (C, D) A 20 -year-old man who showed significant improvement in CMA after surgical release. (E) Cervical scoliosis with 2 curves occurs toward the affected side of CMT. (F) Measurement of LS to assess cervical scoliosis with 2 curves. (G, $\mathrm{H})$ An 8-year-old girl who showed significant improvement in LS after surgical release. (I) Secondary vertebral scoliosis developed to compensate for abnormal posture of head and neck and/or pelvic elevation. (J) Measurement of CA to assess secondary vertebral scoliosis. (K, L) A 9-year-old girl who showed significant improvement in LS after surgical release. CMA, cervicomandibular angle; LS, lateral shift; CA, Cobb angle; CMT, congenital muscular torticollis.

$$
\text { CMA ratio }=\frac{\text { preoperative CMA }- \text { postoperative CMA }}{\text { preoperative CMA }}
$$$$
\text { LS ratio }=\frac{\text { preoperative LS }- \text { postoperative LS }}{\text { preoperative LS }}
$$$$
\text { CA ratio }=\frac{\text { preoperative } \mathrm{CA}-\text { postoperative } \mathrm{CA}}{\text { preoperative } \mathrm{CA}}
$$

\section{Statistical analysis}

Statistical Package for the Social Sciences (SPSS) ver. 19.0 (IBM, Armonk, NY, USA) was used for statistical analysis. Intraclass correlation coefficients (ICCs) between two investigators were calculated for inter-rater reliability. The ICC score was considered reliable if it was $\geq 0.70$. The Wilcoxon signed-rank test was performed to assess the effectiveness of surgical release in relation to treatment of spinal deformities in neglected CMT patients. A linear regression test was performed to assess the correlation between change in spinal deformities after surgical release and age at the time of surgery. Statistical significance was set at $\mathrm{p}<0.05$.

\section{RESULTS}

\section{Characteristics of the subjects}

Forty-six subjects (19 males, 27 females) who met the inclusion criteria were included in this retrospective study. The characteristics of the subjects are presented in Table 1 . The median age at the time surgery was 12.87 years (interquartile range [IQR], 7.58-20.79 years). The CMT of the patients involved the right side in 31 subjects (67.4\%) and the left side in 15 subjects (32.6\%) (Table 1). A total of 46 subjects had undergone plain AP radi- 
ography of the cervical spine, once before surgery, and again between 6 and 15 months after surgery. CMA was measured from plain AP radiographs of the cervical spine in 45 subjects. We also measured LS and CA from 27 subjects who underwent plain AP radiography of the whole spine.

Quantitative analysis of AP plain radiographs and interrater reliability

Quantitative analysis of spinal deformities measured by two investigators is presented in Table 2 along with the

Table 1. Subject characteristics $(n=46)$

\begin{tabular}{lc}
\hline \multicolumn{1}{c}{ Characteristic } & Value \\
\hline $\begin{array}{l}\text { No. of subjects who underwent } \\
\text { cervical-spine radiography }\end{array}$ & 45 \\
$\begin{array}{l}\text { No. of subjects who underwent } \\
\text { whole-spine radiography }\end{array}$ & 27 \\
$\begin{array}{l}\text { Age at the time of surgery (yr) } \\
\text { Sex (male:female) }\end{array}$ & $12.87(7.58-20.79)$ \\
$\begin{array}{l}\text { Affected side of CMT affected side } \\
\text { (right:left) }\end{array}$ & $31: 15$ \\
\hline
\end{tabular}

Values are presented as number or median (interquartile range).

CMT, congenital muscular torticollis.
ICC values. All ICC values were $>0.90$, suggesting excellent inter-rater reliability $(\mathrm{p}<0.001)$.

\section{Lateral head tilt measured by CMA}

Significant head tilt $\left(\mathrm{CMA}>10^{\circ}\right)$ was present in $64.4 \%$ of subjects (29 out of 45). An improvement in CMA from surgical release was found in $97.8 \%$ of patients ( 45 out of 46) with neglected CMT.

The postoperative CMA was significantly lower than the preoperative CMA-median $4.02^{\circ}$ (IQR, $2.64^{\circ}-5.97^{\circ}$ ) vs. median $12.13^{\circ}$ (IQR, $7.50^{\circ}-15.86^{\circ}$ ); $\mathrm{p}<0.001$, Wilcoxon signed-rank test (Fig. 3A). The correlation between CMA ratio and age at the time of surgery was not statistically significant $(\mathrm{R}=0.145, \mathrm{p}=0.341$; linear regression analysis) (Fig. 4A).

\section{Cervical scoliosis with 2 curves measured by LS}

Before surgery, a total of 14 subjects (51.9\%) had a significant cervical scoliosis with 2 spinal curves (LS>20 $\mathrm{mm})$. An improvement in LS from surgical release was shown in $88.9 \%$ of patients ( 24 out of 27 ) with neglected CMT.

The postoperative LS was significantly lower than the preoperative LS-median $13.55 \mathrm{~mm}$ (IQR, 8.50-21.37 $\mathrm{mm}$ ) vs. median $18.13 \mathrm{~mm}$ (IQR, 13.83-30.05 $\mathrm{mm}$ );

Table 2. Inter-rater reliability of spinal deformity measurements

\begin{tabular}{|c|c|c|c|c|}
\hline Measurement & Investigator $^{\mathrm{a})}$ & Mean \pm SD & ICC $^{\text {b) }}$ & p-value \\
\hline \multicolumn{5}{|l|}{$\operatorname{CMA}\left({ }^{\circ}\right)$} \\
\hline \multirow[t]{2}{*}{ Preoperative } & 1 & $12.83 \pm 6.55$ & 0.97 & $<0.001$ \\
\hline & 2 & $12.78 \pm 6.60$ & & \\
\hline \multirow[t]{2}{*}{ Postoperative } & 1 & $4.63 \pm 3.53$ & 0.97 & $<0.001$ \\
\hline & 2 & $4.82 \pm 3.50$ & & \\
\hline \multicolumn{5}{|l|}{$\mathrm{LS}(\mathrm{mm})$} \\
\hline \multirow[t]{2}{*}{ Preoperative } & 1 & $22.12 \pm 15.52$ & 0.96 & $<0.001$ \\
\hline & 2 & $22.93 \pm 15.30$ & & \\
\hline \multirow[t]{2}{*}{ Postoperative } & 1 & $16.64 \pm 14.22$ & 0.96 & $<0.001$ \\
\hline & 2 & $17.11 \pm 12.76$ & & \\
\hline \multicolumn{5}{|l|}{$\mathrm{CA}\left({ }^{\circ}\right)$} \\
\hline \multirow[t]{2}{*}{ Preoperative } & 1 & $6.84 \pm 3.45$ & 0.91 & $<0.001$ \\
\hline & 2 & $8.15 \pm 3.52$ & & \\
\hline \multirow[t]{2}{*}{ Postoperative } & 1 & $5.60 \pm 3.94$ & 0.92 & $<0.001$ \\
\hline & 2 & $5.86 \pm 3.40$ & & \\
\hline
\end{tabular}

ICC, intraclass correlation coefficient; CMA, cervicomandibular angle; LS, lateral shift; CA, Cobb angle.

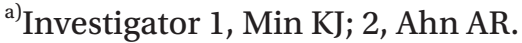

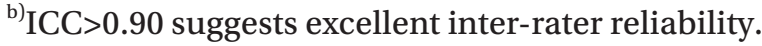


(A)

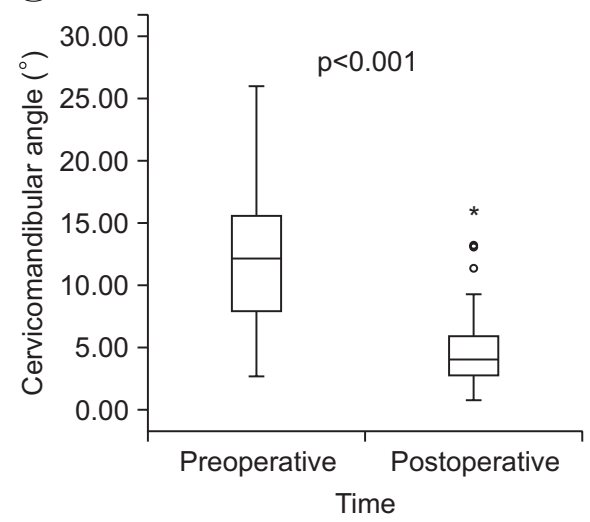

(B)

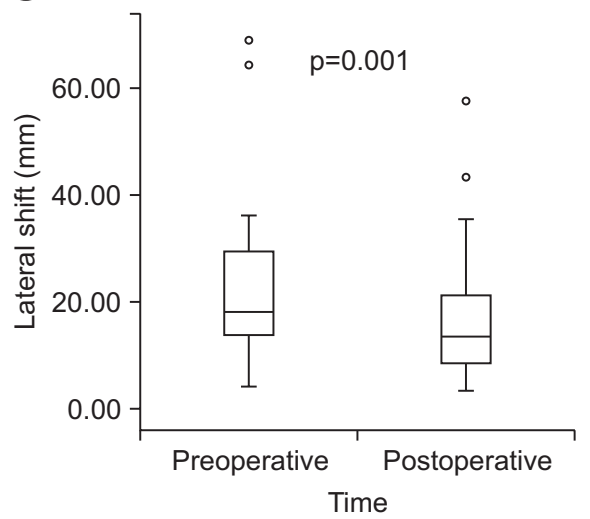

(C)

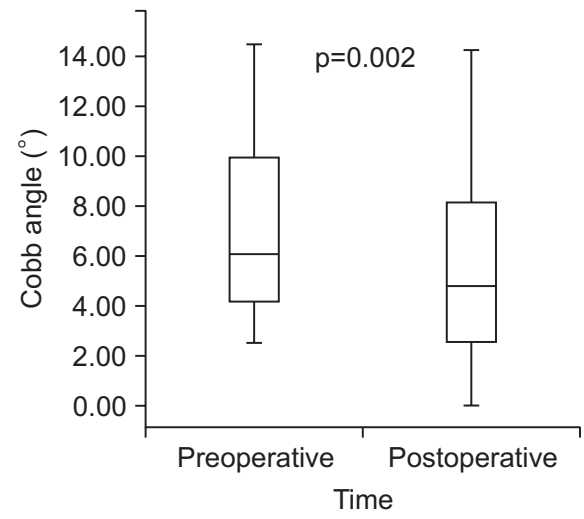

Fig. 3. Effectiveness of surgical release in patients with neglected congenital muscular torticollis. Surgical release resulted in improvements in cervicomandibular angle (A), lateral shift (B), and Cobb angle (C).

(A)

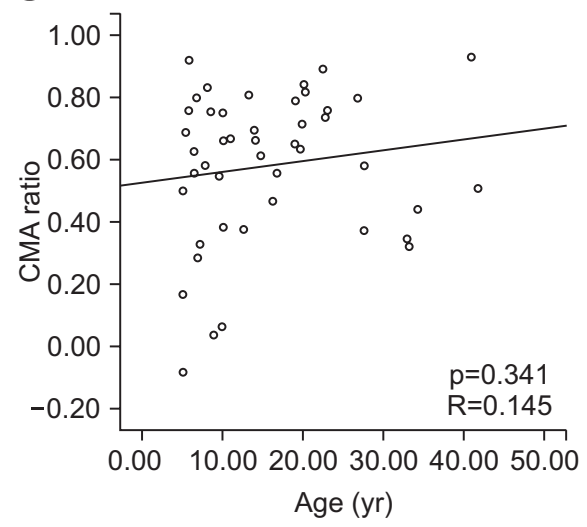

(B)

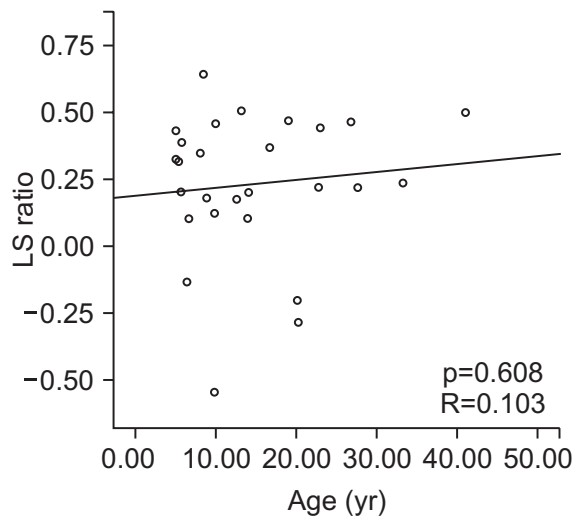

(C)

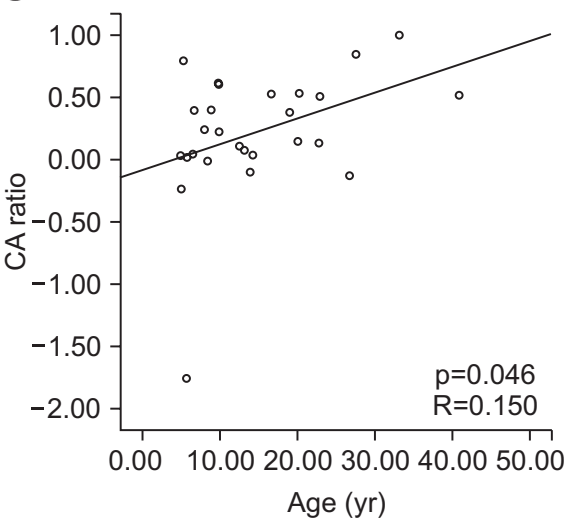

Fig. 4. The correlation between improvement in spinal deformities and age at the time of surgery. The CMA ratio (A) and LS ratio (B) increased slightly with increasing age at the time of surgery. (C) The CA ratio increased significently with increasing age at the time of surgery. CMA, cervicomandibular angle; LS, lateral shift; CA, Cobb angle.

$\mathrm{p}=0.001$, Wilcoxon signed-rank test (Fig. 3B). The correlation between lateral shift ratio and age at the time of surgery was not statistically significant $(R=0.103, p=0.608$; linear regression analysis) (Fig. 4B).

\section{Vertebral scoliosis measured by CA}

Before surgery, 7 subjects (25.9\%) had significant vertebral scoliosis $\left(\mathrm{CA}>10^{\circ}\right)$. An improvement in CA from surgical release was shown in $77.8 \%$ of patients ( 21 out of 27) with neglected CMT.

The postoperative CA was significantly lower than the preoperative CA-median $4.80^{\circ}$ (IQR, $2.41^{\circ}-8.87^{\circ}$ ) vs. median $6.10^{\circ}$ (IQR, $3.93^{\circ}-10.11^{\circ}$ ); $\mathrm{p}=0.002$, Wilcoxon signedrank test (Fig. 3C). There was a statistically significant positive correlation between CA ratio and age at the time of surgery ( $\mathrm{R}=0.150, \mathrm{p}=0.046$; linear regression analysis) (Fig. 4C).

\section{DISCUSSION}

To our knowledge, for patients with neglected CMT, this study is the first to assess the effectiveness of surgical release for spinal deformities according to age at the time of surgery. We used imaging techniques such as neck MRI and ultrasonography to diagnose CMT. Ultrasonography is usually used as a diagnostic tool for CMT in neonates or infants when subjects have a significant difference in the thicknesses of each SCM muscle [9]. After remis- 
sion of swelling of the affected SCM, even if atrophic or hyperechogenic changes of SCM muscle are found, their presence alone is not sufficient to confirm the diagnosis. Moreover, if subjects are hesitant to undergo surgery, MRI is used to determine the necessity for operation [21]. Because most of our subjects were diagnosed after 5 years of age and could be operated upon, MRI was used mainly for diagnosis of CMT, with all enrolled patients undergoing MRI before surgical release. However, for subjects who visited a clinic when they were infants, ultrasonography was performed for diagnosis. Thus, we used the imaging modalities of ultrasonography and MRI of the neck to determine clinical criteria for enrollment.

A previous study reported that surgical release in adults with neglected CMT led to cosmetic and functional improvements as assessed using plain radiographs and questionnaires [6]. The authors identified cosmetic improvements after surgical release by analyzing plain radiographs of the cervical and whole spine obtained from 37 patients with neglected CMT between 18 and 48 years; however, they did not analyze the relationship between the effectiveness of surgical release and age at the time of surgery.

Our results indicate that, in patients with neglected CMT, the effectiveness of surgical release for spinal deformities does not decrease with increasing age at the time of surgery. Our result can establish another basis for the timing of surgery for patients with neglected CMT in that invasive surgical release can reduce spinal deformities even in older patients. However, in contrast to our expectation that there would be no significant difference in improvement according to age, the improvement in vertebral scoliosis measured by CA increased significantly with increasing age at the time of surgery. This might be due to higher rates of compliance with postoperative exercises for scoliosis in older patients since adults tend to be more concerned about scoliosis than children. However, a larger study is required to accurately evaluate the correlation between secondary vertebral scoliosis and timing of surgery in patients with neglected CMT.

There was an improvement among all 3 parameters of spinal deformity after surgery, and no surgical complications were reported. Previous studies reported optimal results when patients underwent surgery between the ages of 1 and 4 years [11-13], and there has been some debate over the pros and cons of the effectiveness and necessity of surgical release in patients with neglected CMT $[13,14]$. Our results suggest that patients with neglected CMT who meet the clinical criteria for surgical release should undergo surgery, and this could be another basis for surgical release guidelines for patients with neglected CMT.

Our study revealed that, before surgery, $25.9 \%$ of patients (7 out of 27) with neglected CMT showed significant secondary vertebral scoliosis $\left(\mathrm{CA}>10^{\circ}\right)$. In the current literature, the overall prevalence of adolescent idiopathic scoliosis is $0.47 \%$ to $5.2 \%$ [22]. Our results demonstrate that secondary vertebral scoliosis is more often present in patients with neglected CMT than in subjects without CMT. A larger study is needed to determine the prevalence of secondary scoliosis in patients with neglected CMT.

According to our previous meta-analysis, which included 12 studies and 220 patients with neglected CMT, there was no significant difference in surgical outcomes between patients older than 15 years and patients 15 years and younger [15]. The range of motion of the neck and skeletal deformities also showed significant improvement with surgical release in this meta-analysis. Although not previously mentioned, we performed a subgroup analysis with an older group (age $>15$ years) and a younger group (age $\leq 15$ years), and significant improvement in CMA and LS was shown in both groups, although CA was only significantly improved in the older group. These results are in agreement with those of our previous meta-analysis, demonstrating that invasive interventions such as surgical release of the affected SCM are required even in neglected CMT patients over 15 years of age.

This study has several strengths. Few reports have investigated the effectiveness of surgical release in patients with neglected CMT $[6,23]$ or the progression of complications such as pain, craniofacial asymmetry, and skeletal deformities along with aging $[6,8,12]$. However, our study is the first to investigate the effectiveness of surgical release for spinal deformities and its correlation with age in patients with neglected CMT.

Second, plain radiography is an objective method used to quantitatively assess the degree of spinal deformities in CMT. Although measuring the degree of tilting or rotation of the head and neck with a goniometer is broadly used to assess skeletal deformities in CMT, it is difficult to measure this in a blinded way because visible surgical 
scars are a potential pitfall in that they make they reveal to the investigators whether the patients underwent surgical treatment or not. Therefore, we assessed spinal deformities using an objective method.

The other strength of the current investigation was that we controlled the risk of AP plain radiography measurement bias. Two authors assessed the AP plain radiographs in a blinded and independent fashion. Since the interrater reliability as verified by an ICC assessment was sufficiently high, the mean value of the two observers was used.

This study has several study limitations. First, this was a retrospective study, which inherently contains the possibility of selection bias. Second, this study included only 46 subjects. Although we found that surgical release was effective in patients with neglected CMT and proved that this effectiveness does not decline with age (against our expectation), the improvement in CA increased with age. Greater compliance with scoliosis exercises in older subjects is postulated as the cause; however, since more subjects could lead to more precise results, a larger study is necessary. Third, even though all patients performed rehabilitation exercises of consistent protocol, the duration of rehabilitation therapy was different for each subject because treatment was ended when the full range of motion of neck rotation was obtained. The varying duration of rehabilitation therapy could affect the changes in spinal deformities after surgical release. However, in this study, the duration of rehabilitation therapy was not assessed for any link to age at the time of surgery. Therefore, further studies should evaluate the effect of rehabilitation protocol factors such as duration on the relationship between age at the time of surgery and effectiveness of surgical release in terms of spinal deformities.

Finally, although all of the patients included in this study were resistant to physiotherapy and had undergone surgical release, there is still the chance that their condition could have spontaneously improved without surgical release.

In conclusion, based on a quantitative evaluation of AP plain radiographs, the improvement of spinal deformities (that is, lateral head tilt, 2-curve cervical scoliosis, and vertebral scoliosis) assessed using CMA, LS, and CA did not decrease with increasing age at the time of surgery. The effectiveness of surgical release for spinal deformities was significant. Despite the several limitations of this study, these findings enhance our understanding of the effectiveness of surgical release in terms of secondary spinal deformities in patients with neglected CMT. Furthermore, we suggest that surgical release be considered even in patients of advanced age with neglected CMT.

\section{CONFLICT OF INTEREST}

No potential conflict of interest relevant to this article was reported.

\section{REFERENCES}

1. Cheng JC, Wong MW, Tang SP, Chen TM, Shum SL, Wong EM. Clinical determinants of the outcome of manual stretching in the treatment of congenital muscular torticollis in infants: a prospective study of eight hundred and twenty-one cases. J Bone Joint Surg Am 2001;83:679-87.

2. Chen MM, Chang HC, Hsieh CF, Yen MF, Chen TH. Predictive model for congenital muscular torticollis: analysis of 1021 infants with sonography. Arch Phys Med Rehabil 2005;86:2199-203.

3. Cheng JC, Tang SP, Chen TM, Wong MW, Wong EM. The clinical presentation and outcome of treatment of congenital muscular torticollis in infants: a study of 1,086 cases. J Pediatr Surg 2000;35:1091-6.

4. Yim SY, Yoon D, Park MC, Lee IJ, Kim JH, Lee MA, et al. Integrative analysis of congenital muscular torticollis: from gene expression to clinical significance. BMC Med Genomics 2013;6 Suppl 2:S10.

5. Do TT. Congenital muscular torticollis: current concepts and review of treatment. Curr Opin Pediatr 2006;18:26-9.

6. Lim KS, Shim JS, Lee YS. Is sternocleidomastoid muscle release effective in adults with neglected congenital muscular torticollis? Clin Orthop Relat Res 2014;472:1271-8.

7. Kuo AA, Tritasavit S, Graham JM Jr. Congenital muscular torticollis and positional plagiocephaly. Pediatr Rev 2014;35:79-87.

8. Jeong KY, Min KJ, Woo J, Yim SY. Craniofacial asymmetry in adults with neglected congenital muscular torticollis. Ann Rehabil Med 2015;39:440-50.

9. Yim SY, Lee IY, Cho KH, Kim JK, Lee IJ, Park MC. The laryngeal cough reflex in congenital muscular tor- 
ticollis: is it a new finding? Am J Phys Med Rehabil 2010;89:147-52.

10. Yim SY, Lee IY, Park MC, Kim JH. Differential diagnosis and management of abnormal posture of the head and neck. J Korean Med Assoc 2009;52:705-18.

11. Canale ST, Griffin DW, Hubbard CN. Congenital muscular torticollis: a long-term follow-up. J Bone Joint Surg Am 1982;64:810-6.

12. Hollier L, Kim J, Grayson BH, McCarthy JG. Congenital muscular torticollis and the associated craniofacial changes. Plast Reconstr Surg 2000;105:827-35.

13. Ling CM. The influence of age on the results of open sternomastoid tenotomy in muscular torticollis. Clin Orthop Relat Res 1976;(116):142-8.

14. Tse P, Cheng J, Chow Y, Leung PC. Surgery for neglected congenital torticollis. Acta Orthop Scand 1987;58:270-2.

15. Kim HJ, Ahn HS, Yim SY. Effectiveness of surgical treatment for neglected congenital muscular torticollis: a systematic review and meta-analysis. Plast Reconstr Surg 2015;136:67e-77e.

16. Hwang JH, Lee HB, Kim JH, Park MC, Kwack KS, Han JD, et al. Magnetic resonance imaging as a determinant for surgical release of congenital muscular torti- collis: correlation with the histopathologic findings. Ann Rehabil Med 2012;36:320-7.

17. Lee SJ, Han JD, Lee HB, Hwang JH, Kim SY, Park MC, et al. Comparison of clinical severity of congenital muscular torticollis based on the method of child birth. Ann Rehabil Med 2011;35:641-7.

18. Shim JS, Noh KC, Park SJ. Treatment of congenital muscular torticollis in patients older than 8 years. J Pediatr Orthop 2004;24:683-8.

19. Shim JS, Jang HP. Operative treatment of congenital torticollis. J Bone Joint Surg Br 2008;90:934-9.

20. Cobb JR. Outline for the study of scoliosis. Instr Course Lect 1948;5:261-75.

21. Kim JW, Kim SH, Yim SY. Quantitative analysis of magnetic resonance imaging of the neck and its usefulness in management of congenital muscular torticollis. Ann Rehabil Med 2015;39:294-302.

22. Konieczny MR, Senyurt H, Krauspe R. Epidemiology of adolescent idiopathic scoliosis. J Child Orthop 2013;7:3-9.

23. Omidi-Kashani F, Hasankhani EG, Sharifi R, Mazlumi M. Is surgery recommended in adults with neglected congenital muscular torticollis? A prospective study. BMC Musculoskelet Disord 2008;9:158. 